\title{
Primera cita del género Melikaiella (Hym.: Cynipidae) para Colombia y descripción de una especie nueva
}

\author{
Juli Pujade-Villar \\ Avda. Diagonal, 645. 08028 Barcelona \\ jpujade@ub.edu

\section{Pedro A. Rodríguez} \\ ICA - Instituto Colombiano Agropecuario \\ Tibaitatá, kilómetro 14 vía Bogotá, Mosquera, Colombia \\ pedro.rodriguez@ica.gov.co
}

Universitat de Barcelona. Facultat de Biologia. Departament de Biologia Animal

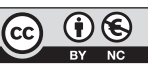

Fecha de recepción: 11 de septiembre de 2015

Fecha de aceptación: 16 de septiembre de 2015

Fecha de publicación: 24 de noviembre de 2015

\section{Resumen}

Se describe una nueva especie del género Melikaiella para Colombia: M. humboldti n. sp. Esta especie corresponde a una forma agámica que produce agallas en bellotas de pequeño tamaño sobre $Q$. humboldtii. Se exponen e ilustran los caracteres morfológicos que definen esta nueva especie. El género Melikaiella se menciona por primera vez para Colombia y representa la distribución más al sur del hemisferio norte conocida del género.

Palabras clave: Hymenoptera; Cynipoidea; Cynipini; Melikaiella; nueva especie; Q. humboldtii; Colombia.

\begin{abstract}
First record of Melikaiella genus (Hym.: Cynipidae) from Colombia and description of a new species

A new species of Melikaiella genus is described from Colombia: M. humboldti n. sp. This species correspond to agamic form producing galls in small acorns of $Q$. humboldtii. Morphological characters defining this new species are exposed and illustrated. Melikaiella is mentioned for the first time in Colombia and represent the further meridional distribution of this genus.
\end{abstract}

Keywords: Hymenoptera; Cynipoidea; Cynipini; Melikaiella; new species; Q. humboldtii; Colombia. 


\section{Introducción}

La familia Cynipidae (Hymenoptera: Cynipoidea) agrupa casi en su totalidad especies de fitófagos, ya sean inductores de agallas o inquilinas de éstas. De acuerdo con Ronquist et al. (2015), se diferencian en 12 tribus: Aylacini, Aulacideini, Ceroptresini, Cynipini, Diastrophini, Diplolepidini, Eschatocerini, Paraulacini, Pediaspidini, Phanacidini, Qwaqwaini y Synergini. En Colombia han sido citadas 5 especies de Cynipidae: Diastrophus colombianus Nieves-Aldrey, 2013 (Diastrophini; Nieves-Aldrey et al., 2013), Synergus colombianus Nieves-Aldrey, 2005 (Synergini; Nieves-Aldrey, 2005) y, Zapatella nievesaldreyi Melika \& Pujade-Villar, 2012, Z. inflata Pujade-Villar \& Rodríguez, 2015 y Z. tuberosa PujadeVillar \& Caicedo (Cynipini; Pujade-Villar et al., 2012; Pujade-Villar et al., 2015).

El género Melikaiella Pujade-Villar, 2014 fue erigido (Pujade-Villar et al., 2014) para incluir diversas especies americanas del género Callirhytis mal ubicadas; de esta forma se incorporaron a Melikaiella una especie mexicana [M. sonorae (Weld, 1944)] y 7 de Estados Unidos [M. corrugis (Bassett, 1881), M. flora (Weld, 1922), M. fructicola (Ashmead, 1897), M. fructuosa (Weld, 1922), M. lupana (Weld, 1944), M. tumifica (Osten-Sacken, 1865) y M. papula (Bassett, 1881)]; y además 4 especies nuevas [3 de México (M. amphibolensis Pujade-Villar, 2014; M. bicolor Pujade-Villar, 2014, M. reticulata Pujade-Villar, 2014) y 1 de EEUU (M. ostensackeni Pujade-Villar, 2014)]. Su distribución por tanto es americana, estando presente tanto en la zona Holártica como en la Neotropical. La especie que aquí se describe representa la cita más al sur del género.

Melikaiella se caracteriza por presentar surco malar, una carena circumscutelar y terguitos metasomales con escultura aerolada. Por otra parte, Melikaiella se caracteriza por tener: una escultura de coriácea a rugosa en el mesoescudo, metaescutelo rugoso-reticulado, fosetas metanotales y laterales del propodeo con una pubescencia densa, carenas irradiantes del clípeo presentes a lo sumo a lo largo del surco malar y siempre ausentes en la zona de la cara inferior hasta toruli, uñas tarsales simples, las hembras con el margen alar sin pubescencia y espina ventral del hipopigio corta provista de un penacho de setas terminal.

Las nueva especie que se describe presenta como hospedador un roble rojo: Q. humboltii Bonpl. (sección Lobatae). Quercus humboldtii es una especie endémica de Colombia y está catalogada, en el libro rojo de plantas maderables de Colombia, como vulnerable (Cardenas \& Salinas, 2007). Presenta una amplia distribución en la zona andina, dominando en las vertientes internas de las tres cordilleras, entre los 1.600 y los 3.000 m.s.n.m. (Mabel-Paz, 2012).

\section{Material y métodos}

Los adultos fueron obtenidos a partir de bellotas colectadas en $Q$. humboldtii. Los insectos emergieron de las bellotas conservadas en cajas de cría en condiciones ambientales de laboratorio.

Para la terminología de las estructuras morfológicas de los adultos se han seguido los estudios de Liljeblad \& Ronquist (1998) y Melika (2006), para la nervia- 
ción alar a Ronquist \& Nordlander (1989) y para la escultura cuticular a Harris (1979). Las mediciones y abreviaturas utilizadas son: F1-F12, primero y siguientes flagelómeros; POL (distancia post-ocelar) es la distancia entre los márgenes interiores de los ocelos posteriores; OOL (distancia ocelar-ocular) es la distancia desde el borde exterior de un ocelo posterior y el margen interno del ojo compuesto; LOL (distancia lateral-ocelar), es la distancia entre ocelos lateral y frontal. La anchura de la celda radial ala anterior se mide desde el margen del ala hasta la vena Rs.

Las imágenes fueron tomadas por el primer autor con un microscopio electrónico ambiental de barrido (FEI Quanta 200 ESEM) en el Servicio CientíficoTécnico de la Universidad de Barcelona (UB, Cataluña), a bajo voltaje y sin recubrimiento de oro; el habitus fue tomado utilizando una cámara Leica DFC450 acoplada a una lupa binocular Leica MZ160A y combinándose 22 fotografías con el programa de apilamiento y procesamiento de imágenes Helicon Focus 6.2.2, mientras que la agalla fue tomada directamente con una cámara digital Canon (Power Shot SX 210/15).

El material tipo ha sido depositado en las siguientes instituciones (entre paréntesis se indican los responsables):

- IAvH Instituto Alexander von Humboldt, Villa de Leyva, Colombia (Claudia Medina).

- UB Universitat de Barcelona, Catalunya (col. Juli Pujade-Villar).

\section{Resultados}

\section{Melikaiella humboldti n. sp.}

Material tipo. HOLOTIPO $q$ (depositado en IAvH): «Borde de Carretera del municipio de Arcabuco, departamento de Boyacá, Colombia), 2.618 m., 0544'59,8"N 73²5'59,9"W, Q. humboldtii, (15-I-2011) 17-I-2011. leg. P.A. Rodríguez» (etiqueta blanca), «Holotype of Melikaiella himboldti ǒ n. sp., design. JP-V 2015» (etiqueta roja). PARATIPOS (1ǒ) con los mismos datos que el holotipo (depositado en UB, col. JP-V).

Diagnosis. La forma agámica que se describe en éste estudio, Melikaiella humboldti n. sp., acerca morfológicamente a las hembras de la forma sexual de $M$. bicolor, ya que ambas especies presentan en común: color ambarino con aéreas negras más o menos extensas, surco malar con carenas irradiantes desde el clípeo, pronoto lateralmente con carenas paralelas, mesoescudo no jorobado medialmente en visión lateral notaulos completos, mesoescudo reticulado con alginas carenas o arrugas, mesoescutelo rugoso, Rs visible aunque débilmente pigmentada, carenas del propodeo delimitando un área esculturada carenado-rugosa y segundo terguito metasomal liso. Ambas hembras se diferencian por los siguientes caracteres: antenas con 12 flagelómeros, el F1 a lo sumo es 2,3 veces más largo que el pedicelo (11 flagelómeros y F1 más de 3,0 veces más largo que ancho en M. humboldti), pronoto fuertemente rugoso lateralmente (débilmente en $M$. humboldti), 
mesoescutelo emarginado lateralmente (carena marginal solo visible en los laterales $M$. humboldti), primer par de alas sin areola (presente in $M$. humboldti), agallas en las ramas (en las bellotas $M$. humboldti).

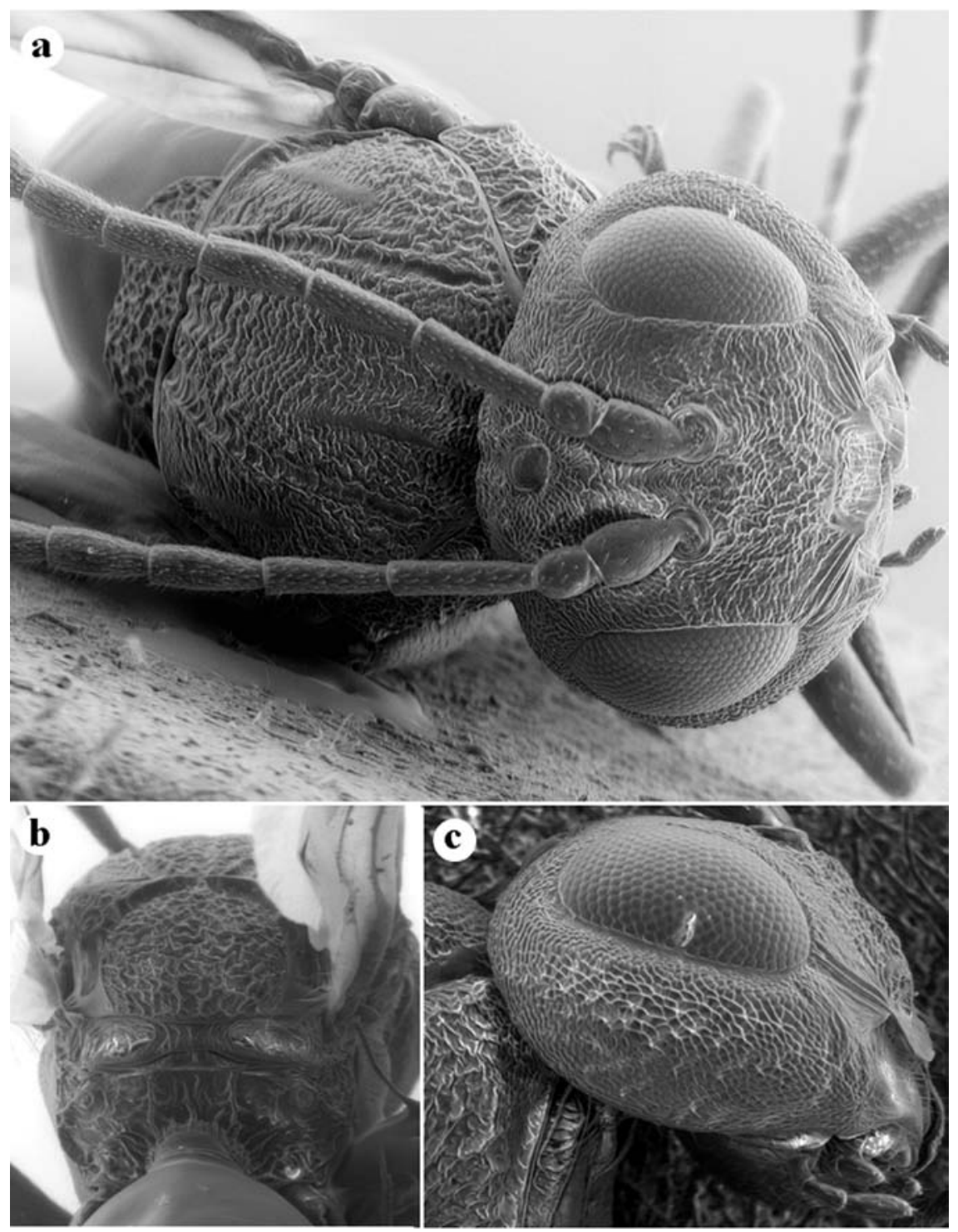

Figura 1. Figuras al microscopio electrónico ambiental de barrido de Melikaiella humboldti: (a) cabeza y mesosoma en visión dorso-anterior, (b) propodeo y, (c) cabeza en visión lateral. 
Descripción. Forma asexual.
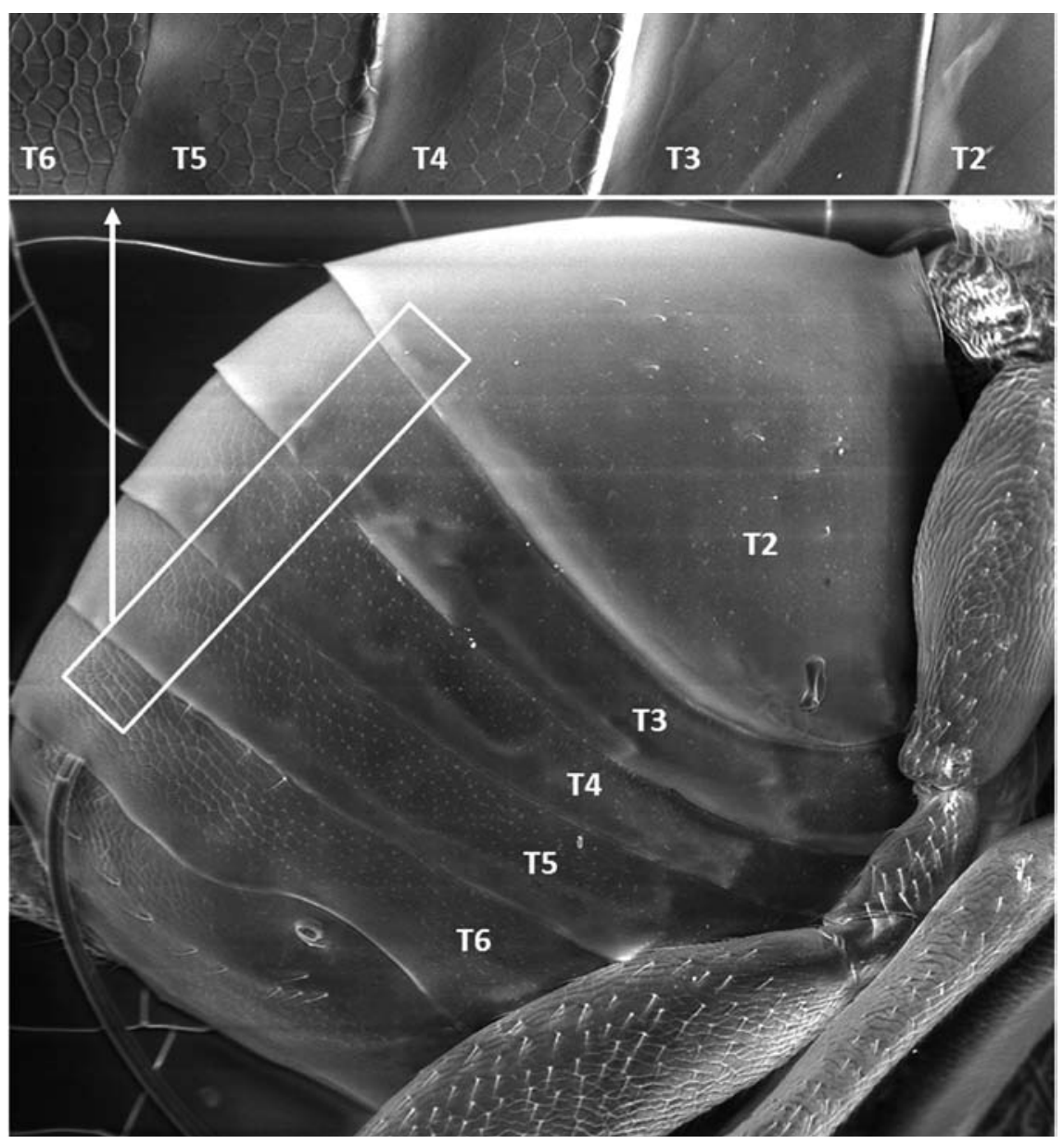

Figura 2. Figura al microscopio electrónico ambiental de barrido del metasoma en visión lateral de Melikaiella humboldti.

Longitud. Hembra: 2,2-2,4 $\mathrm{mm}(\mathrm{n}=2)$.

Coloración (Fig. 3b). Ambarina; antenas y patas más claras, amarillentas; escutelo negro; mesopleura castaña, más clara inferiormente; propodeo oscuro, de castaño a negro; metasoma marrón. Alas con una tonalidad amarillenta o transparente, venas muy poco pigmentadas. 


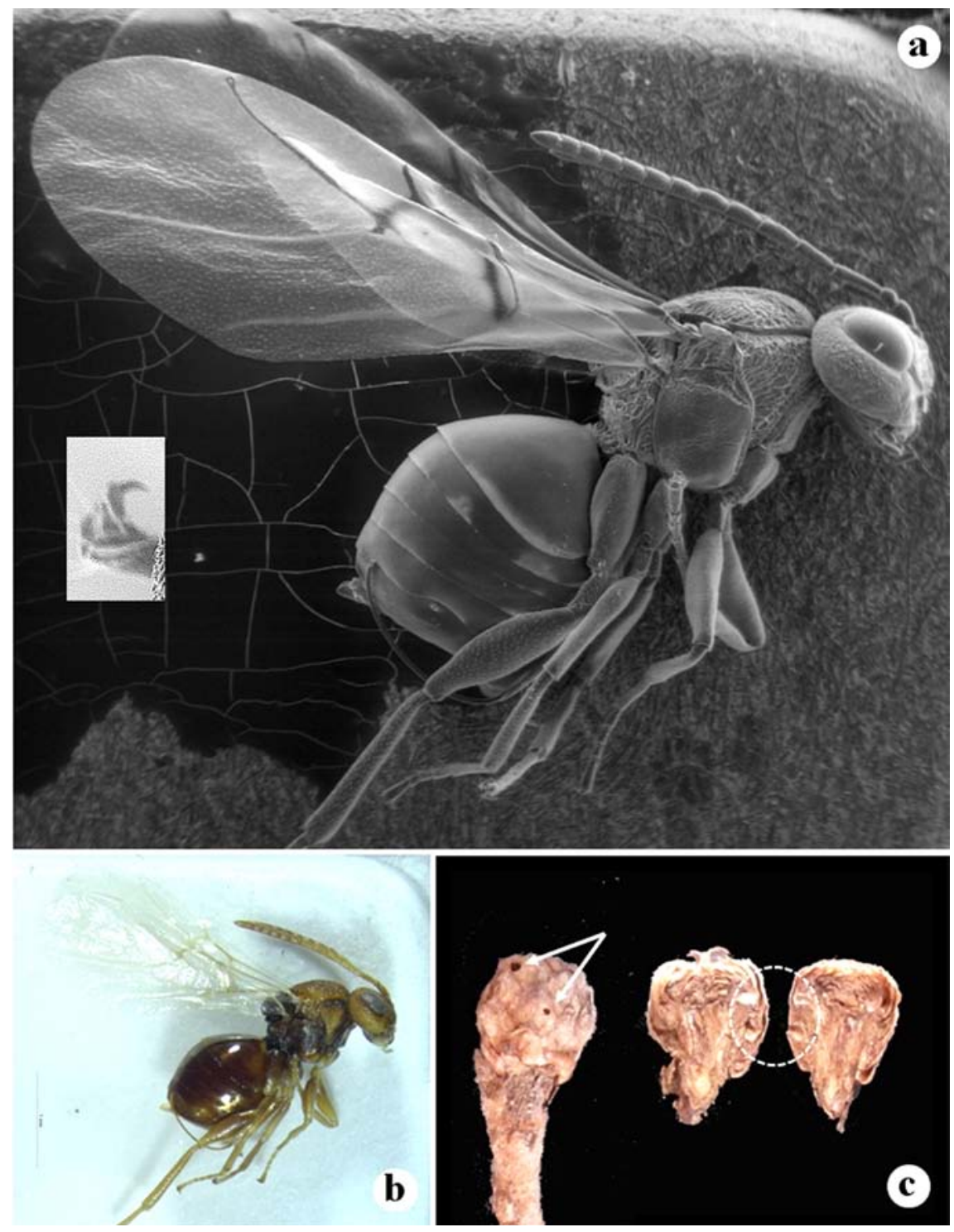

Figura 3. Melikaiella humboldti: (a) imagen del habitus al microscopio electrónico ambiental de barrido y detalle de la uña tarsal, (b) imagen digital y, (c) agallas indicando con flechas los agujeros de salida en la bellota y bellota cortada indicado las cámaras larvales.

Cabeza (Figs. 1a-b). Ligeramente más ancha que el mesosoma, con escasa setas de color blanquecino, cortas, poco visibles. En visión frontal oval transversal, 1,4 
veces tan ancha como alta y transversal desde arriba, unas 2,4 veces más ancha que larga en vista dorsal; gena amplia detrás del ojo, lateralmente 1,5 veces más ancha que el diámetro transversal del ojo, con una escultura reticulado-imbricada; espacio malar con surco, 0,4 veces más largo que la altura del ojo compuesto, con algunas estrías visibles que irradian de clípeo dispuestas alrededor del surco malar casi llegando casi al margen ojo. Cara inferior coriácea, zona medial elevada. Clípeo marcado, pubescencia poco evidente, coriáceo en el centro y ligeramente emarginado ventralmente; margen distal liso y débilmente inciso, fosetas tentoriales pequeñas, poco visibles; surco epistomal y la línea clípeo-pleurostomal visibles. POL:OOL:OOL = 5:4:3, OOL con una longitud similar al doble del diámetro del ocelo lateral, área interocelar fuertemente coriácea, no elevada; frente y vértice fuertemente coriáceos; occipucio coriáceo. Palpo labial 3- trisegmentado, escasamente pubescente; palpo maxilar 5-segmentado, los cuatro segmentos terminales escasamente pubescentes.

Antena (Figs. 1a, 3a). 13 segmentos (a veces sutura incompleta en el F11), ligeramente más larga que la longitud combinada de cabeza y mesosoma (casi 1,4 veces), pero mucho más corta que la longitud del cuerpo ( 0,7 veces). F3-F11 más gruesos que F1 y F2; pedicelo globoso, tan largo como ancho; F1 más largo que F2 y siguientes decreciendo en longitud, F10 escasamente más largo que ancho; F11 2,0 veces más largo, como F10 (a veces con dos subunidades visibles siendo la distal un poco más larga que a inferior); sensilas placodeas en F3-F11, escasas en F3-F4. Fórmula antenal: 4: 2: $7: 6: 5: 4,5: 4: 3: 3: 3: 3: 2,5: 2,5+3$.

Mesosoma (Figs. 1a-b, 3a). Ligeramente más largo que alto, con el mesoescudo cóncavo dorsalmente en vista lateral. Pronoto prácticamente glabro, con una escultura lateral coriácea-reticulada, con carenas visibles posterolateralmente. Mesoescudo ligeramente más ancho que largo en vista dorsal, prácticamente glabro, que contrastan muy poco con la coloración del escudo; escultura fuertemente reticulada con algunas carenas o arrugas más visibles en los laterales. Notaulos completos, profundos, más amplios posteriormente, fondo alutáceo; línea mesoscutal mediana presente pero indistinta; líneas parapsidales diferenciadas, coriáceas, que se extienden más allá del nivel de las tégulas; líneas paralelas anteriores distintas, coriáceas, extendiéndose hasta en nivel de las tégulas. Mesopleura uniformemente coriáceo-reticulado. Mesoescutelo un poco más largo que ancho en vista dorsal, uniformemente rugoso; fóveas escutelares estrechas, ovales, con fondo débilmente esculturado (alutáceo) provisto de algunas carenas débiles y brillantes, separadas medialmente por un punto. Metaescutelo coriáceo, más alto que la altura de la zona lisa y brillante impresionada ventralmente al metanoto; foseta metanotal coriácea-alutácea, brillante, pubescente. Propodeo fuertemente alutáceo, con escasas setas blancas lateralmente; área propodeal brillante, centro alutáceo con carenas o arrugas visibles, glabro, delimitado por las carenas laterales subparalelas. Nucha corta, con carenas longitudinales. 
Patas (Fig. 3a). Uñas tarsales simples, sin lóbulo basal ni base amplia; área dorsoposterior de las coxas posteriores desprovistas de densas setas blancas.

Primer par de alas (Fig. 3a). Más largas que la longitud del cuerpo (1,2-1,3 veces), pubescentes con setas cortas, sin cilios en los márgenes; celda radial abierta, alrededor de 3,8 veces más larga que ancha; venas muy poco pigmentadas, casi no trazables; areola visible a contraluz; vena Rs + M dirigiéndose ligeramente por debajo de la mitad de la vena basal; R1 y Rs casi alcanzando el margen de ala, muy poco visibles.

Metasoma (Figs. 2, 3a). Tan largo como la cabeza y mesosoma juntos, ligeramente más largo que alto; segundo terguito metasomal liso y brillante, glabro. Tercer terguito metasomal dorsalmente con escultura alutácea, liso y brillante lateralmente; terguitos siguientes alutáceos. Espina ventral del hipopigio corta (un poco más corta que larga), con setas apicales formando un penacho.

Agallas (Fig. 3c). Completamente inconspícuas, situadas en el interior de los frutos, en pequeñas bellotas. Las cámaras larvales están situadas perpendicularmente en la parte leñosa de la cúpula; son pequeñas y ovales $(1,4 \times 0,7 \mathrm{~mm})$ con la pared fina, frágil y de color amarillo.

Biología. Sólo hembras se obtienen de las cámaras larvales internas de pequeñas bellotas de $Q$. humboldtii. Las bellotas se colectaron en enero y las avispas adultas emergieron en el mismo mes pocos días después de la recolección.

Distribución. Conocida solo de Colombia, departamento de Boyacá, en el municipio de Arcabuco en bosques mixtos latifoliados de hoja caduca, ubicados por encima de los $2.000 \mathrm{~m}$ de altitud.

Etimología. El nombre específico hace referencia a su hospedador, el roble Colombiano

\section{Discusión}

Callirhytis fue erigido por Förster (1869) para incluir varias especies europeas, cuyos principales caracteres diagnósticos genéricos eran tener el mesoescudo estriado transversalmente y presentar surco malar. Weld (1922a, 1922b, 1926, 1952) incluye muchas especies del Neártico en el género Callirhytis, descuidando los caracteres diagnósticos establecidos por Förster (1869). El resultado fue que $\mathrm{Ca}$ llirhytis 'sensu Weld' se convirtió en un género caótico. Melika \& Abrahamson (2002) mencionan que, de las 115 especies que se citan en el Neártico (Burks, 1979), solo unas 15 de ellas pertenecen al género Callirhytis en dicha región biogeográfica. Estudios posteriores han transferido algunas especies al género Andricus Hartig, 1840 (ver Pujade-Villar et al., 2013) o bien a géneros recientemente descritos que producen agallas en la sección Lobatae: Zapatella (ver Pujade-Vi- 


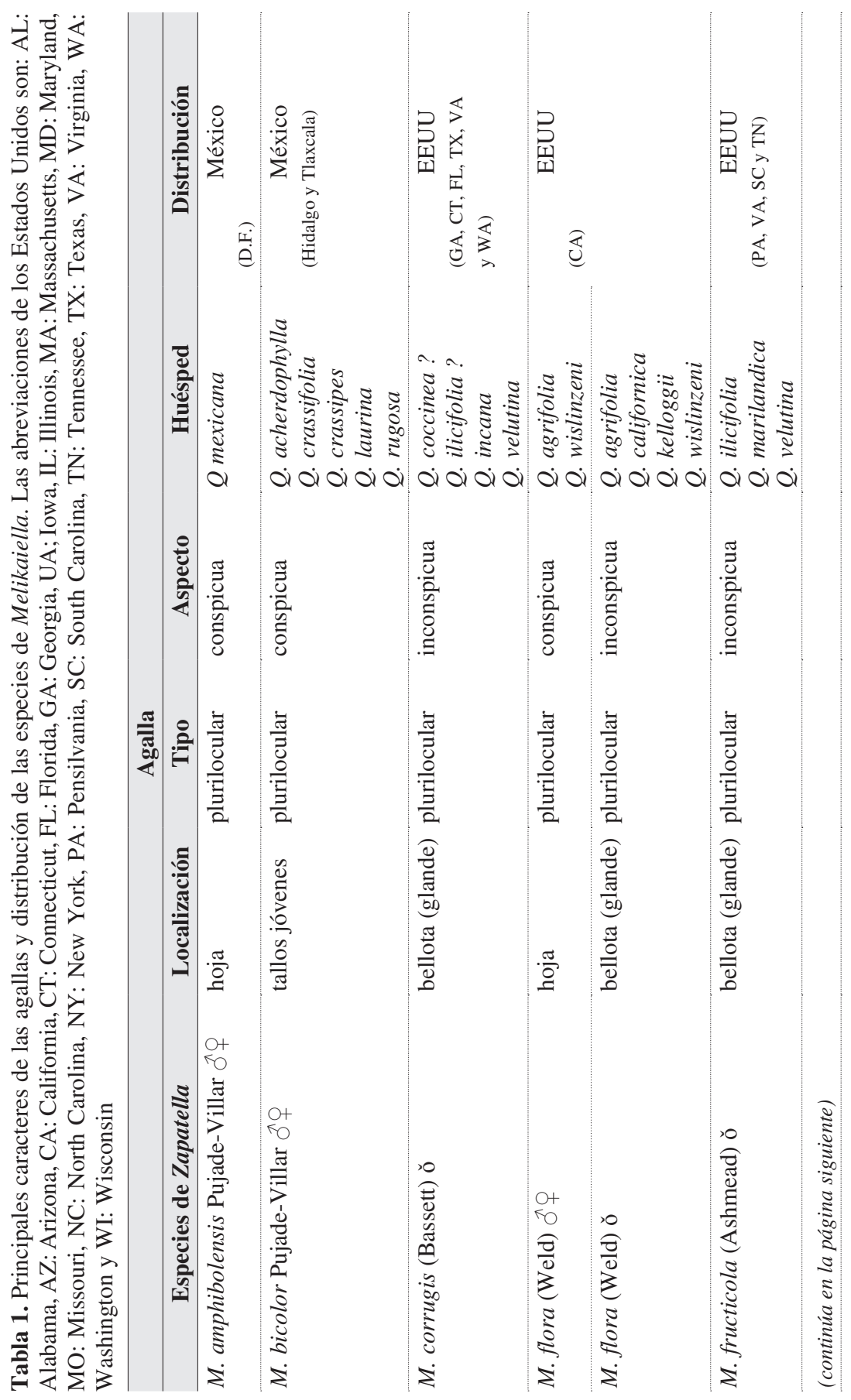




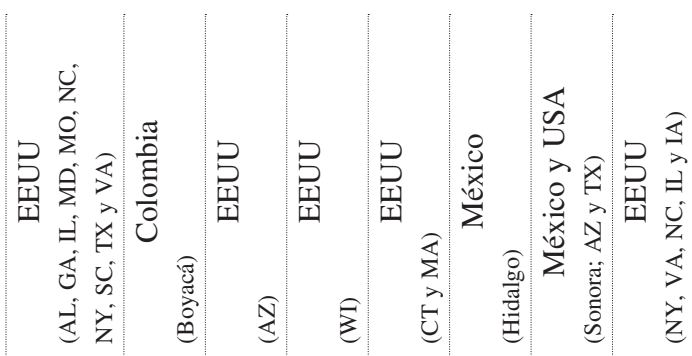

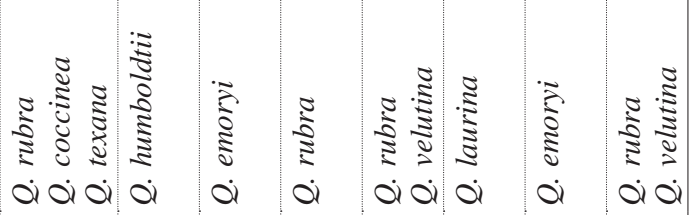

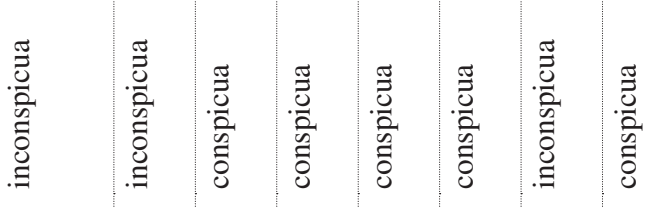

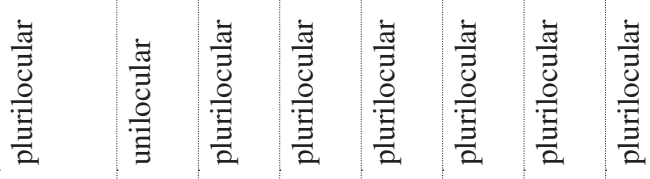

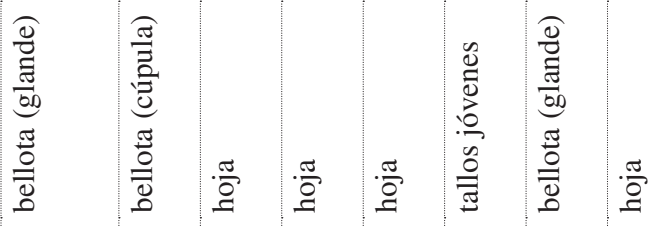

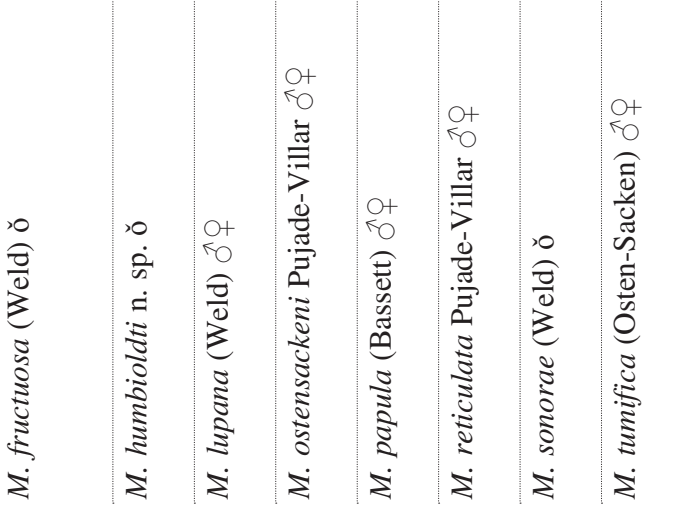


llar et al., 2012) y Melikaella (ver Pujade-Villar et al., 2012); Callirhytis en el Paleártico produce agallas en la sección Quercus, en el Holártico está por comprobar.

Con los resultados de este estudio, Melikaiella, que presenta una distribución americana, está presente tanto en la región Holártica como Neotropical. En la actualidad, el género agrupa 13 especies (Tabla 1). Casi todas las formas sexuadas producen agallas en las hojas, concretamente corresponden a agallas pluriloculares que deforman la nerviación principal (Tabla 1) y las formas agámicas se encuentran en su mayor parte en los glandes de las bellotas desarrolladas. La especie nueva que aquí se describe es la única que se encuentra en bellotas jóvenes y la única que presenta las cámaras larvales en el tejido leñoso de la cúpula de la bellota.

\section{Agradecimientos}

Agradecemos muy sinceramente a nuestro colega Marcos Roca-Cusachs (UB) el haber realizado la fotografía del habitus y a los dos evaluadores anónimos del manuscrito que han contribuido a mejorar el resultado final.

\section{Referencias bibliográficas}

Burks, B.D. 1979. Superfamily Cynipoidea. In: Krombein, K.V., Hurd, P.D., Jr., Smith, D.R.; Burks, B.D. (Eds.). Catalog of Hymenoptera in America North of Mexico, Vol. 1, Symphyta and Apocrita, pp. 1045-1107, Smithsonian Institution Press, Washington, DC. 1198 pp.

Cardenas, L.D.; Salinas, N.R. 2007. Libro rojo de plantas de Colombia, Volumen 4, especies maderables amenazadas, primera parte. Instituto Amazónico de Investigaciones Científicas SINCHI. Ministerio de Ambiente, Vivienda y Desarrollo Territorial. $232 \mathrm{pp}$.

Förster, A. 1869. Ueber die Gallwespen. Verh. Zool.-Bot. Gesselschaft Wien 19: 327-370.

Harris, R. 1979. A glossary of surface sculpturing. State of California, department of food and agriculture. Occasional Papers Entomol. 28: 1-31.

Liljeblad, J.; Ronquist, F. 1998. A phylogenetic analysis of higher-level gall wasp relationships (Hymenoptera: Cynipidae). Syst. Entomol. 23: 229-252.

Melika, G., 2006. Gall Wasps of Ukraine. Cynipidae. Vestnik zoologii, supplement 21(12): 1-300, 301-644.

Melika, G.; Abrahamson, W.G. 2002. Review of the World Genera of Oak Cynipid Wasps (Hymenoptera: Cynipidae: Cynipini). In: Melika, G.; Thuróczy, C. (Eds.). Parasitic Wasps: Evolution, Systematics, Biodiversity and Biological Control, pp. 150-190, Agroinform, Budapest. $480 \mathrm{pp}$.

Nieves-Aldrey, J.L. 2005. Notes on the Neotropical species of Synergus Hartig (Hymenoptera, Cynipidae), including the description of a new species from Colombia. Canadian Entomologist 137: 501-508.

Nieves-Aldrey, J.L.; Rodríguez, P.A.; Medianero, E. 2013. Description of a new species of Diastrophus (Hymenoptera: Cynipidae: «Aylacini») from Colombia: the first herb gall wasp native to the Neotropical Region. Annals of the Entomological Society of America, 106(6): 719-728. 
Mabel Paz, P.G. 2012. Variabilidad genética del roble común (Quercus humboldtii Bonpl.) en la región del macizo colombiano. Biotec. Sector Agropec. Agroind. 10 (2): 110-116.

Pujade-Villar, J. 2013. Las agallas de los encinos: un ecosistema en miniatura que hace posible estudios multidisciplinares. Entomol. Mexicana 21(1): 2-22.

Pujade-Villar, J.; Hanson, P.; Medina, C.A.; Torres, M.; Melika, G. 2012. A new genus of oak gall wasps, Zapatella Pujade-Villar \& Melika, gen. n., with a description of two new species from the Neotropics (Hymenoptera, Cynipidae, Cynipini). ZooKeys 210: 75-104.

Pujade-Villar, J.; Pérez-García, A.G.; Equihua-Martínez, A.; Estrada-Venegas, E.G.; Cibrián-Tovar, D.; Barrera-Ruíz, U.M.; Ferrer-Suay, M. 2013. Review of Andricus species (Hym., Cynipidae) producing woody tuberous oak galls in Mexico and bordering areas of United States. Dugesiana 20(2): 183-208.

Pujade-Villar, J.; Rodríguez, P.A.; Caicedo, G. 2015. Dos nuevas especies de Zapatella (Hym., Cynipidae) para Colombia que producen agallas en ramas de Quercus humboldtii (Fagaceae). Butll. Inst. Catalana Hist. Nat. 79: 79-90.

Ronquist, F.; Nordlander, G. 1989. Skeletal morphology of an archaic cynipoid, Ibalia rufipes (Hymenoptera: Ibaliidae). Entomol. Scandinavica Suppl. 33: 1-60.

Ronquist, F.; Nieves-Aldrey, J.L.; Buffington, M.L.; Liu, Z.; Liljeblad, J.; Nylander, J.A.A. 2015. Phylogeny, Evolution and Classification of Gall Wasps: The Plot Thickens. The Plot Thickens. PLoS ONE 10(5): e0123301. $<$ http://dx.doi.org/10.1371/journal.pone.0123301>

Weld, L.H. 1922a. Notes on Cynipid wasps, with descriptions of new North American species. Proc. United States Nat. Mus. 61(18): 1-29.

Weld, L.H. 1922b. Notes on American gallflies of the family Cynipidae producing galls on acorns, with descriptions of new species. Proc. United States Nat. Mus. 61(19): 1-23.

Weld, L.H. 1926. Field Notes on Gall-inhabiting Cynipid Wasps with descriptions of new species. Proc. United States Nat. Mus. 68(10): 1-131.

Weld, L.H. 1952. Cynipoidea (Hym.) 1905-1950 being a Supplement to the Dalla Torre and Kieffer monograph, the Cynipidae in Das Tierreich, Leiferung 24, 1910 and bringing the systematic literature of the world up to date, including keys to families and subfamilies and list of new generic, specific and variety names. Ann Arbor, Michigan. Privately printed. $351 \mathrm{pp}$. 